\title{
EDITORIAL
}

\section{An internationally recognized uniform cytogenetic classification system is needed for multiple myeloma}

\section{Leukemia (2007) 21, 9-11. doi:10.1038/sj.leu.2404450}

In this issue of Leukemia there are 3 papers $^{1-3}$ dealing with the prognostic impact of abnormal cytogenetics in patients with multiple myeloma. Two address whether bortezomib overcomes the poor outcomes associated with deletion $13,{ }^{1,2}$ whereas the final one evaluates the role different fluorescent in situ hybridization (FISH) abnormalities play in the prognosis of patients undergoing autologous hematopoietic stem cell transplantation (HSCT). ${ }^{3}$

As background, the reader should be aware of two important concepts and a controversy. The first is the distinction between deletion of chromosome 13 by metaphase cytogenetics $(M-\Delta 13)$ and by interphase FISH (F- $\Delta 13) .{ }^{3-6} \mathrm{M}-\Delta 13$ is found in only $15 \%$ of all newly diagnosed myeloma patients, ${ }^{7-10}$ whereas $\mathrm{F}-\Delta 13$ is found in approximately $50 \%$ of the same population. ${ }^{3,11,12}$ This discrepancy is because the former technique is reliant on proliferating myeloma cells to generate a result, whereas the latter is independent of cell division. The second concept is a derivative of the first: $M-\Delta 13$ is a composite prognostic marker because it provides information about both proliferation and the chromosome 13 structural abnormality. ${ }^{13}$ The controversy revolves around the point that although $M-\Delta 13$ is universally accepted as an adverse prognostic factor, ${ }^{6,7}$ the extent deletion $\mathrm{F}-\Delta 13$ plays as an independent adverse prognostic factor is in question. ${ }^{9,14}$

The paper by Jagannath et al. ${ }^{1}$ explores whether bortezomib can overcome the adverse impact of deletion 13 using patients' data from the SUMMIT and APEX trials, two trials studying the role of bortezomib in relapsed/refractory myeloma patients. Although together, the two trials included 535 patients receiving bortezomib, only 221 and 102 had evaluable metaphase cytogenetics or FISH studies, respectively. In retrospective matched pair analyses using a fraction of these patients, the authors found that bortezomib provided outcomes (response rates, time to progression and overall survival) in $M-\Delta 13$ patients comparable to those patients without $M-\Delta 13$ in both trials (Table 1). In contrast, patients enrolled on the APEX trial, who received single-agent dexamethasone and had $M-\Delta 13$, did significantly poorer than those without the abnormality. At first blush, the relative equivalence seen in patients with and without $M-\Delta 13$ could be brushed aside by the possibility that this merely reflects a type II error - that is, the sample size and duration of follow-up are just too short to reveal the statistical difference between patients with and without $M-\Delta 13$ who received bortezomib. This criticism, however, is partially mitigated by the profound difference seen in dexamethasone-treated patients with and without $M-\Delta 13$. Even with the limitations of small sample size and short follow-up, outcomes associated with the presence of $M-\Delta 13$ in the dexamethasone-treated patients were far worse than in its absence. So, based on these M- $\Delta 13$ data, one can speculate that even if bortezomib does not completely level the playing field for $M-\Delta 13$ patients, it appears to reduce partially the disadvantage. The authors appropriately concede that their results need to be confirmed by others.
A twist to the Jagannath et al.'s paper arises when the same case-matched analyses were performed by using FISH in the presence or absence of deletion 13. Again, there was no significant difference in outcome for those bortezomib-treated patients with and without F- $\Delta 13$; however, no difference was seen in dexamethasone-treated patients with and without F- $\Delta 13$ either! How does one reconcile the differences between the M- $\Delta 13$ and F- $\Delta 13$ analyses in the Jagannath et al.'s study? Their FISH results would appear to challenge the argument offered in defense of the $M-\Delta 13$ findings. Perhaps, the difference can be explained, in part by the differential prognostic impact $F-\Delta 13$ has as compared to $M-\Delta 13$, that is, proliferation - or perhaps, these inconsistencies are all a function of small sample size.

Moreover, the paper by Sagaster et al. ${ }^{2}$ in this issue supports the findings of Jagganath et al., in that Sagaster et al. also find that overall response and time to treatment failure are not different in bortezomib-treated patients regardless of deletion 13 status (Table 1); however, in this latter study, there was a trend toward inferior survival in the $\mathrm{F}-\Delta 13$ patients as compared to those without the abnormality. In addition, Sagaster et al. make the provocative observation that all three patients with $t(4 ; 14)$ responded to bortezomib and they propose a risk classification system incorporating deletion 13 and serum albumin. A major caveat to this study is that the patients with $\mathrm{F}-\Delta 13$ may have had other prognostic factors that influenced their overall outcomes. Take for example, the fact that the time from diagnosis was only 26 months for the F- $\Delta 13$ patients in contrast to 51 months for the patients without the deletion. Is this imbalance because patients with $\mathrm{F}-\Delta 13$ have shorter times to progression and therefore have exhausted other treatments more quickly, or is it due to the fact that the group of $\mathrm{F}-\Delta 13$ patients who responded to bortezomib were earlier along in their disease course? These are questions that cannot be answered by this small retrospective review. A recent publication by Mateos et al. ${ }^{16}$ also bolsters the favorable influence bortezomib may have on F- $\Delta 13$ patients. With 16month follow-up, they reported that newly diagnosed, elderly myeloma patients treated with VMP demonstrated no difference in PFS for those patients with and without F- $\Delta 13$ (Table 1).

The final paper in this issue of Leukemia that deals with cytogenetic abnormalities in myeloma patients is that of Gutiérrez et al. ${ }^{2}$ These authors analyze the FISH findings of 260 newly diagnosed patients participating in an HSCT trial and scrutinize the role $\mathrm{F}-\Delta 13$ plays as a prognostic factor in patients with other FISH abnormalities. The authors probed for retinoblastoma gene deletion (standard surrogate for $\mathrm{F}-\Delta 13$ ), chromosome 14 translocations and the p53 deletion $\mathrm{F}-\Delta 17 \mathrm{p} 51$. Their analysis is a complicated one, with multiple comparisons and sub-analyses. The authors report that F- $\Delta 13, \mathrm{~F}-\Delta 17 \mathrm{p} 51$ and $14 q 32$ translocations had significant adverse impact on time to progression and overall survival on univariate analysis, but go on to further dissect the role other abnormalities have on $\mathrm{F}-\Delta 13^{\prime} \mathrm{s}$ impact on survival by whittling the 109 patients with $\mathrm{F}-\Delta 13$ down to 46 patients without any of the other FISH abnormalities (14q32 translocations or F- $\Delta 17 \mathrm{p} 51)$ and report that median overall survival for these 46 patients was not 
Table 1 Effect of bortezomib therapy on patients with deletion $13^{1,2,15}$

\begin{tabular}{|c|c|c|c|c|c|c|}
\hline Treatment & Abnormality & $\mathrm{N}$ & $\pi P(m o)$ & $P$-value & MS (mo) & $P$-value \\
\hline Bortez-SUMMIT ${ }^{1}$ & $M-\Delta 13$ & 26 & 2.6 & NS & 10 & NS \\
\hline Bortez -SUMMIT ${ }^{1}$ & No $M-\Delta 13$ & 26 & 3.3 & - & NR & - \\
\hline Bortez -APEX ${ }^{1}$ & $M-\Delta 13$ & 64 & 2.6 & 0.06 & 12.5 & 0.8 \\
\hline Bortez -APEX ${ }^{1}$ & No $M-\Delta 13$ & 64 & 7.7 & - & $\mathrm{NR}$ & - \\
\hline $\operatorname{Dex}^{1}$ & $M-\Delta 13$ & 61 & 2.8 & 0.2 & 3.3 & 0.002 \\
\hline Dex $^{1}$ & No $M-\Delta 13$ & 61 & 5.6 & - & NR & - \\
\hline Bortez -APEX'1 & $F-\Delta 13$ & 18 & 6.2 & 0.2 & NR & $\mathrm{NE}$ \\
\hline Bortez -APEX ${ }^{1}$ & No $F-\Delta 13$ & 18 & 4.6 & - & NR & - \\
\hline $\operatorname{Dex}^{1}$ & $\mathrm{~F}-\Delta 13$ & 20 & 3.5 & 0.6 & NR & $\mathrm{NE}$ \\
\hline $\operatorname{Dex}^{1}$ & No $F-\Delta 13$ & 20 & 2.8 & - & NR & - \\
\hline Bortez $^{2}$ & $\mathrm{~F}-\Delta 13$ & 33 & $4.6^{a}$ & 0.95 & 9.9 & 0.057 \\
\hline Bortez $^{2}$ & No $F-\Delta 13$ & 29 & $6.7^{\mathrm{a}}$ & & NR & \\
\hline VMP - newly Dx ${ }^{15}$ & $F-\Delta 13$ & 13 & $92 \%$ at 1 year & NS & NP & - \\
\hline VMP - newly Dx ${ }^{15}$ & No $F-\Delta 13$ & 20 & $91 \%$ at 1 year & - & NP & - \\
\hline
\end{tabular}

Bortez, bortezomib; Dex, dexamethasone; F- $\Delta 13$, deletion 13 by FISH; M- $\Delta 13$, deletion 13 by metaphase cytogenetics; mo, months; MS, median overall survival; NE, not evaluable; NP, not provided; NR, not reached; NS, not significant; TTP, time to progression; VMP, bortezomib, melphalan, prednisone.

aTime to treatment failure.

significantly different than the overall survival of the 109 patients without any FISH abnormality (54 versus 46 months). This is an interesting finding, but the authors' conclusion that ' $\mathrm{Rb}$ deletion as a unique abnormality is not associated with adverse prognosis', is too strong given the limitations of their data. The median follow-up for these patients is only 34 months, and, therefore, conclusions about portions of survival curves distant from median follow-up should be tempered, especially when there are pre-existing data from larger studies that show contradictory findings. Earlier this year, in this journal, Chiecchio et al. ${ }^{6}$ studied 729 patients by FISH, $81 \%$ of whom were newly diagnosed. They reported that the median overall survivals of patients with different FISH abnormalities were significantly different: no F- $\Delta 13$, median overall survival not reached; F- $\Delta 13$ as the only abnormality, 29 months; F- $\Delta 13$ with either an $14 q 32$ translocation or F $\Delta 17$ p51, 20 months; and $\mathrm{F}-\Delta 13$ with both an $14 \mathrm{q} 32$ translocation and $\mathrm{F} \Delta 17 \mathrm{p} 51,13$ months. Chiecchio et al.'s study also has limitations, that is, no treatment information and a median follow-up of only 22 months.

So what can we conclude from these three studies in this issue of Leukemia? They provide important hypothesis generating ammunition. Based on the data of Jagannath et al and Sagaster et al., $^{2}$ we need to further test the hypothesis that bortezomib may have a favorable influence on patients with $M-\Delta 13$, which may be a function of deletion of the retinoblastoma gene and/or a function of the proliferative index of the myeloma cells. The Gutiérrez data provides us with confirmation that $t(4 ; 14), 17 \mathrm{p} 51$ and F- $\Delta 13$ carry significant adverse prognostic influence in patients undergoing stem cell transplantation. $., 8,16-19$

These studies also underline the fact that a uniform, practical cytogenetic staging system is needed and should be defined by the myeloma community. Although the International Staging System, ${ }^{20}$ which uses beta- 2 microglobulin and albumin, has been a major step forward in the direction of 'speaking the same prognostic language', the myeloma community is ready for more. Already it has been shown repeatedly that in patients with multiple myeloma, deletion 13 is the most common monosomy, $^{21}$ that there is a strong association between it and $14 q 32$ translocations, ${ }^{12,22}$ that $\mathrm{t}(4 ; 14)$ is among the worst-recognized translocations ${ }^{17-19,23}$ and that deletion $17 \mathrm{p} 51$ also has prognostic implications. ${ }^{8,17,24-26}$ Perhaps a similar meeting of the minds as used for the International Staging System could be arranged to finalize a practical, internationally accepted cytogenetic staging system that can be used in clinical practice.

At present, what are the implications for the clinician taking care of a myeloma patient? All newly diagnosed patients (and all clinical trial patients) should have both a standard metaphase karyotypic analysis performed as well as a standard myeloma FISH panel. Only in this way will we be able to best educate our current patients about their individual prognosis and to understand the role novel agents and combinations play in overcoming the adverse prognostic effect of possessing an adverse cytogenetic feature for future generations of myeloma patients.

A Dispenzieri Mayo Clinic, Rochester, MN, USA E-mail: dispenzieri.angela@mayo.edu

\section{References}

1 Jagannath S, Richardson PG, Sonneveld P, Schuster MW, Irwin D Stadtmauer EA et al. Bortezomib appears to overcome the poor prognosis conferred by chromosome 13 deletion in phase 2 and 3 trials. Leukemia 2006.

2 Gutiérrez NC, Castellanos MV, Martin ML, Mateos MV, Hernandez JM, Fernandez $M$ et al. Prognostic and biological implications of genetic abnormalities in multiple myeloma undergoing autologous stem cell transplantation: $t(4 ; 14)$ is the most relevant adverse prognostic factor, while RB deletion as a unique abnormality is not associated with adverse prognosis. Leukemia 2006.

3 Kaufmann H, Kromer E, Nosslinger T, Weltermann A, Ackermann $\mathrm{J}$, Reisner $\mathrm{R}$ et al. Both chromosome 13 abnormalities by metaphase cytogenetics and deletion of $13 q$ by interphase FISH only are prognostically relevant in multiple myeloma. Eur J Haematol 2003; 71: 179-183.

4 Shaughnessy Jr J, Tian E, Sawyer J, McCoy J, Tricot G, Jacobson J et al. Prognostic impact of cytogenetic and interphase fluorescence 
in situ hybridization-defined chromosome 13 deletion in multiple myeloma: early results of total therapy II. Br J Haematol 2003; 120 : 44-52.

5 Dewald GW, Therneau T, Larson D, Lee YK, Fink S, Smoley S et al. Relationship of patient survival and chromosome anomalies detected in metaphase and/or interphase cells at diagnosis of myeloma. Blood 2005; 106: 3553-3558.

6 Chiecchio L, Protheroe RK, Ibrahim AH, Cheung KL, Rudduck C, Dagrada GP et al. Deletion of chromosome 13 detected by conventional cytogenetics is a critical prognostic factor in myeloma. Leukemia 2006; 20: 1610-1617.

7 Tricot G, Barlogie B, Jagannath S, Bracy D, Mattox S, Vesole DH et al. Poor prognosis in multiple myeloma is associated only with partial or complete deletions of chromosome 13 or abnormalities involving $11 \mathrm{q}$ and not with other karyotype abnormalities. Blood 1995; 86: 4250-4256.

8 Konigsberg R, Zojer N, Ackermann J, Kromer E, Kittler H, Fritz E et al. Predictive role of interphase cytogenetics for survival of patients with multiple myeloma. J Clin Oncol 2000; 18: 804-812.

9 Smadja NV, Bastard C, Brigaudeau C, Leroux D, Fruchart C, Groupe Francais de Cytogenetique $\mathrm{H}$. Hypodiploidy is a major prognostic factor in multiple myeloma. Blood 2001; 98 2229-2238.

10 Fonseca R, Harrington D, Oken MM, Dewald GW, Bailey RJ, Van Wier SA et al. Biological and prognostic significance of interphase fluorescence in situ hybridization detection of chromosome 13 abnormalities (delta13) in multiple myeloma: an eastern cooperative oncology group study. Cancer Res 2002; 62: 715-720.

11 Dao DD, Sawyer JR, Epstein J, Hoover RG, Barlogie B, Tricot G. Deletion of the retinoblastoma gene in multiple myeloma. Leukemia 1994; 8: 1280-1284.

12 Avet-Loiseau H, Facon T, Grosbois B, Magrangeas F, Rapp MJ, Harousseau JL et al. Oncogenesis of multiple myeloma: $14 \mathrm{q} 32$ and $13 \mathrm{q}$ chromosomal abnormalities are not randomly distributed, but correlate with natural history, immunological features, and clinical presentation. Blood 2002; 99: 2185-2191.

13 Rajkumar SV, Fonseca R, Dewald GW, Therneau TM, Lacy MQ, Kyle RA et al. Cytogenetic abnormalities correlate with the plasma cell labeling index and extent of bone marrow involvement in myeloma. Cancer Genet Cytogenet 1999; 113: 73-77.

14 Stewart AK, Fonseca R. Prognostic and therapeutic significance of myeloma genetics and gene expression profiling. J Clin Onco 2005; 23: 6339-6344.

15 Mateos MV, Hernandez JM, Hernandez MT, Gutierrez NC, Palomera L, Fuertes $\mathrm{M}$ et al. Bortezomib plus melphalan and prednisone in elderly untreated patients with multiple myeloma: results of a multicenter phase I/II study. Blood 2006; 108: 2165-2172.

16 Moreau P, Facon T, Leleu X, Morineau N, Huyghe P, Harousseau JL et al. Recurrent 14q32 translocations determine the prognosis of multiple myeloma, especially in patients receiving intensive chemotherapy. Blood 2002; 100: 1579-1583.

17 Gertz MA, Lacy MQ, Dispenzieri A, Greipp PR, Litzow MR, Henderson KJ et al. Clinical implications of $t(11 ; 14)(q 13 ; q 32)$, $\mathrm{t}(4 ; 14)(\mathrm{p} 16.3 ; \mathrm{q} 32)$, and $-17 \mathrm{p} 13$ in myeloma patients treated with high-dose therapy. Blood 2005; 106: 2837-2840.

18 Chang H, Sloan S, Li D, Zhuang L, Yi QL, Chen Cl et al. The t $(4 ; 14)$ is associated with poor prognosis in myeloma patients undergoing autologous stem cell transplant. $\mathrm{Br}$ J Haematol 2004; 125: 64-68.

19 Cavo M, Terragna C, Renzulli M, Zamagni E, Tosi P, Testoni N et al. Poor outcome with front-line autologous transplantation in $\mathrm{t}(4 ; 14)$ multiple myeloma: low complete remission rate and short duration of remission. J Clin Oncol 2006; 24: e4-e5.

20 Greipp PR, San Miguel J, Durie BG, Crowley JJ, Barlogie B, Blade J et al. International staging system for multiple myeloma. J Clin Oncol 2005; 23: 3412-3420.

21 Debes-Marun CS, Dewald GW, Bryant S, Picken E, Santana-Davila $\mathrm{R}$, Gonzalez-Paz $\mathrm{N}$ et al. Chromosome abnormalities clustering and its implications for pathogenesis and prognosis in myeloma. Leukemia 2003; 17: 427-436.

22 Fonesca R, Oken MM, Greipp PR, Eastern Cooperative Oncology Group Myeloma G. The $t(4 ; 14)(p 16.3 q 32)$ is strongly associated with chromosome 13 abnormalities in both multiple myeloma and monoclonal gammopathy of undetermined significance. Blood 2001; 98: 1271-1272.

23 Avet-Loiseau H, Li JY, Facon T, Brigaudeau C, Morineau N, Maloisel $\mathrm{F}$ et al. High incidence of translocations $\mathrm{t}(11 ; 14)(\mathrm{q} 13 ; \mathrm{q} 32)$ and $\mathrm{t}(4 ; 14)(\mathrm{p} 16 ; \mathrm{q} 32)$ in patients with plasma cell malignancies. Cancer Res 1998; 58: 5640-5645.

24 Chang H, Qi C, Yi QL, Reece D, Stewart AK. p53 gene deletion detected by fluorescence in situ hybridization is an adverse prognostic factor for patients with multiple myeloma following autologous stem cell transplantation. Blood 2005; 105: 358-360.

25 Drach J, Ackermann J, Fritz E, Kromer E, Schuster R, Gisslinger H et al. Presence of a p53 gene deletion in patients with multiple myeloma predicts for short survival after conventional-dose chemotherapy. Blood 1998; 92: 802-809.

26 Chng WJ, Santana-Davila R, Van Wier SA, Ahmann GJ, Jalal SM, Bergsagel PL et al. Prognostic factors for hyperdiploid-myeloma: effects of chromosome 13 deletions and $\mathrm{IgH}$ translocations. Leukemia 2006; 20: 807-813. 